\title{
Superantigènes viraux
}

Les superantigènes sont des antigènes particuliers au contact desquels les manif estations immunologiques présentent une ampleur exceptionnelle. Ils sont caractérisés par une forte expansion polyclonale engendrant une production massive de cytokines suivie, dans la plupart des cas, par l'apparition d'un état de non-réponse. L'installation d'un état de non-réponse consécutif à la stimulation superantigénique serait interprétée comme l'engagement du lymphocyte sur une voie d'activation incomplète. Ces propriétés résultent d'une association particulière des superantigènes avec les dimères $\alpha / \beta$ des molécules du complexe majeur d'histocompatibilité (CMH) de classe II et avec la chaîne $V_{\beta} d u$ récepteur de l'antigène des lymphocytes $T$ (TcR). L'interaction du superantigène avec ces molécules se ferait latéralement et en dehors de la gorge hébergeant le peptide $\left(\mathrm{m} / \mathrm{s} n^{\circ} 1\right.$ (suppl.), vol. 5 , p. 23). La stimulation des cellules $\mathrm{T}$ par le superantigène est indépendante de leur spécificité antigénique, mais est strictement contrôlée par le $V_{\beta}$ exprimé. Elle est, en revanche, faiblement influencée par le polymorphisme des $\mathrm{CMH}$ de classe II. Ces propriétés ont été établies en étudiant les toxines bactériennes, comme les toxines du staphylocoque A, B, ou le TSST-1* qui sont associés à des syndromes de choc toxique et à des effets immunosuppresseurs [1]. Les superantigènes n'ont pas été décrits uniquement chez les bactéries ou les mycoplasmes [2] ; ils l'ont été aussi chez les virus $[3,4]$. L'existence de superantigènes viraux a tout d'abord été établie chez la souris pour les rétro-

* Voir glossaire. virus murins qui induisent des tumeurs des glandes mammaires et modifient l'expression de certaines familles lymphocytaires [4]. Plus récemment, il a été démontré que la nucléocapside du virus de la rage est un superantigène; le champ d'étude des superantigènes viraux s'est vu ainsi élargi au système immunitaire humain [5].

La présence d'un superantigène viral au cours d'infections pose la question de son impact sur le développement de la maladie et sur les maladies du système immunitaire qui leur sont parfois associées.

Propriété prenière d'un superantigène : stinuuler des familles entières de lymphocytes $T$ porteurs de chaînes $V_{\beta}$ données

Une stimulation préférentielle de certaines familles $V_{\beta}$ peut être observée avec des antigènes classiques à épitopes dominants ou avec des peptides (par exemple le virus de la grippe [6] ou le virus de la méningite chorio-lymphocytaire [7]). Mais celle-ci s'accompagne, comme toute réponse antigénique, de la participation d'éléments du TcR autres que la chaîne $V_{\beta}$ (à savoir la chaîne $V_{\alpha}$ et les zones de jonction entre les différents fragments). A la différence, une réponse superantigénique ne fait pas intervenir (ou faiblement) ces éléments. Une réponse superantigénique se distinguera donc d'une réponse antigénique $V \beta$ orientée, par la polyclonalité de la réponse [8]. L'expansion préférentielle polyclonale de certains $V_{\beta}$ est une propriété importante qui laisse suspecter la présence d'un superantigène. C'est celle-ci qui a été recherchée dans les infections causées par le virus d'Epstein-Barr (EBV), l'HTLV-l ou encore le VIH, pour lesquelles on soupçonne la présence d'un superantigène. Dans ces trois cas, l'expansion préférentielle de certains $V_{\beta}$ a été mise en évidence, soit par immunofluorescence, soit par PCR quantitative [9, 10]. Toutefois, l'étude de la polyclonalité des stimulations n'a pas toujours été entreprise. L'expansion intervient peu de temps après le contact avec le superantigène. La recherche d'une stimulation polyclonale aurait, par conséquent, plus de chances d'aboutir en prélevant des cellules à des donneurs qui se trouvent dans les premières phases de l'infection, plutôt qu'à des donneurs en fin d'infection. Le choix de malades en phase initiale ou tardive pourrait être à l'origine des données contradictoires obtenues par différents laboratoires. Pour les rétrovirus murins exogènes, il a été proposé qu'un superantigène puisse favoriser l'infection en stimulant, par l'intermédiaire des lymphocytes $\mathrm{CD}^{+}$, la multiplication des cellules cibles que sont les lymphocytes B [4, 11]. Le superantigène rétroviral murin produit par les cellules infectées interviendrait donc dès les premières étapes du cycle viral. Le superantigène du virus de la rage pourrait, lui aussi, favoriser la multiplication du virus et permettre au virus d'envahir l'hôte, puisqu'il a été établi que les lymphocytes T humains peuvent être infectés par ce virus à condition qu'ils aient été au préalable stimulés. L'influence d'un superantigène dans l'infection par le VlH pourrait être du même ordre. Le virus VIH se multiplie en effet dans les lymphocytes stimulés, avec une préférence pour certains $V_{\beta}$ (par exemple le $V_{\beta 12}$ [12]). Il se pourrait alors que l'effet du superantigène $\mathrm{VlH}$ fút de stimuler les cellules hébergeant et produisant le virus $\mathrm{VIH}[13,14]$. 
Autre propriété: faire disparaître des familles de lymphocytes $T$ porteurs de chaînes $V_{\beta}$ données

Il a été démontré, pour les superantigènes bactériens, que l'imprégnation néonatale avec l'entérotoxine du staphylocoque de type $\mathrm{A}$ induit une délétion néonatale des $V_{\beta}$ spécifiquement reconnus par ce superantigène [15]. L'existence, dans une lignée de souris, de MMTV* intégrés, ou l'infection des souriceaux par allaitement d'une mère infectée par des MMTV* exogènes, se traduit par la disparition de certains $V_{\beta}[4$, 16]. Cette disparition est interprétée comme la conséquence de l'exposition au superantigène pendant la période de maturation du système immunitaire. Le superantigène est alors perçu comme une protéine du soi. Les lymphocytes $T$ stimulés par le superantigène sont éliminés par un mécanisme qui s'apparenterait au phénomène de sélection négative des cellules autoréactives. A la différence de l'établissement de la tolérance à un antigène pour lequel seul un petit nombre de cellules appartenant à différentes familles sont détruites, dans le cas d'un superantigène, ce sont des familles entières de $V_{\beta}$ qui sont éliminées. Leur disparition est donc facilement identifiable par l'analyse des répertoires des lymphocytes thymiques ou périphériques.

L'analyse des répertoires et la recherche de délétions ont été utilisées comme preuves de l'existence d'un superantigène dans l'infection par le VIH. Les résultats des études dans lesquelles on a cherché à mettre en évidence la disparition de $V_{\beta}$ particuliers chez les sujets infectés par le VIH sont contradictoires. Certains groupes concluent à la disparition effective de certains $V_{\beta}$ $\left(\mathrm{V}_{\text {ק917 }}\right)$ [17-19], alors que d'autres groupes n'observent pas de différence significative [14]. Ces travaux ont été entrepris sous l'impulsion de la théorie de Janeway qui proposait d'expliquer les anomalies immunitaires associées à cette infection par l'existence d'un superantigène VIH [20]. Partant de l'observation selon laquelle l'infection des $\mathrm{m} / \mathrm{s} n^{\circ} 1$ vol 10, janviar 94 lymphocytes $\mathrm{CD}^{+}$par le VIH (moins de $1 \%$ des cellules) ne peut expliquer à elle seule la disparition progressive des lymphocytes $\mathrm{CD}^{+}$, Janeway avait proposé qu'elle fût provoquée par la présence d'un superantigène. Ce superantigène stimulerait, dans un premier temps, les farnilles de lymphocytes porteurs des $V_{\beta}$ adéquats, qui, engagés dans une voie d'activation incomplète par le superantigène, seraient par la suite éliminés. Ieur disparition serait la conséquence, soit de l'installation d'un état de non-réponse, soit de l'entrée dans un processus de mort programmée, encore appelée apoptose. La généralisation de la disparition des lymphocytes $\mathrm{CD}^{+}$proviendrait de la grande variabilité du superantigène lié au VIH. La modification du superantigène par mutations progressives induirait la disparition de la majeure partie des lymphocytes recrutés les uns après les autres par les différentes formes de superantigènes. Récemment, il a été établi que le VIH pouvait infecter les lignées hématopoïétiques et, en les détruisant, participer à la destruction de familles entières de $V_{\beta}[13]$. Les macrophages se sont revélés être, eux aussi, des nettoyeurs efficaces de cellules infectées par le VIH [21]. Il n'est donc plus nécessaire de faire appel à l'existence d'un superantigène pour expliquer la disparition des lymphocytes $\mathrm{CD}^{+}[13]$. Toutefois, cette constatation qui affaiblit la portée de la théorie de Janeway ne remet pas en question l'existence d'un superantigène $\mathrm{VIH}$, dont l'activité pourrait être mise en évidence au tout début de l'infection dans sa phase proliférative.

La présence chronique d'un superantigène et les délétions induites pourraient expliquer les complications des vaccinations rougeoleuses à fort titre. Une surmortalité a été récemment mise en évidence au Sénégal, à Haîti, en Guinée-Bissau et au Mexique après des campagnes de vaccination contre la rougeole utilisant, non pas des vaccins atténués classiques, mais des vaccins atténués à fort titre [22]. Une étude menée au Pérou a fait ressortir que les enfants vaccinés avec le vaccin à fort titre, et plus particulièrement les filles, présentaient une altération de leur rapport $\mathrm{CD}^{+} / \mathrm{CD}^{+}$suggérant l'installation d'une immunosuppression retardée. Celle-ci se distinguerait de l'immunosuppression observée lors des phases aiguẽs de rougeole, qui est transitoire et n'est pas marquée par une altération du rapport $\mathrm{CD}^{+} / \mathrm{CD}^{+}$. Les mécanismes d'installation de cette immunosuppresssion sont inconnus. Il est possible qu'elle soit la conséquence d'un effet superantigénique associé à l'établissement d'une infection rougeoleuse chronique.

\section{Nature des superantigènes viraux}

Jusqu'à présent les superantigènes viraux appartiennent à deux catégories. Il s'agit, soit de protéines structurales - le cas de la rage-, soit de protéines non structurales, comme dans le cas des MMTV*.

Le virus rabique est constitué d'une nucléocapside et d'une enveloppe formée de glycoprotéines et de lipides. La nucléocapside résulte de l'association d'un brin d'ARN de polarité négative et des protéines $\mathrm{N}$, NS et L. La protéine $\mathbf{N}$, porteuse des propriétés superantigéniques de la nucléocapside, est une protéine de $55 \mathrm{kDa}$ longue de 450 acides aminés, phosphorylée mais non glycosylée. La nature du superantigène codé par le gène ORF* des MMTV* a été récemment établie. Il s'agit d'une glycoprotéine de type II, de $46 \mathrm{kDa}$, qui ne fait pas partie de la particule virale mais est associée à la cellule infectée [23]. Le transport de cette protéine à la surface des cellules infectées par le MMTV* et son accrochage aux molécules $\mathrm{CMH}$ de classe II sont encore mal définis. Il a été proposé que cette protéine soit hydrolysée puis excrétée, pour être, par la suite, captée par les molécules de CMH de classe II portées à la surface des cellules stimulatrices. La nature de certains superantigènes viraux pourrait être tout autre. Il a ainsi été envisagé que les superantigènes viraux, en particulier dans l'infection par le VIH, puissent ne 
pas être codés par le virus lui-même, mais soient, en fait, des constituants cellulaires modifiés ou produits en quantités anormales au cours de l'infection. Des travaux récents sur le caractère superantigénique du HTLV-l chez le lapin étayent fortement cette hypothèse ([24] et Akira Seto, communication personnelle). Il semblerait ainsi que l'injection de cellules de lapin transformées par le HTLV-l ayant acquis le pouvoir leucémogène stimule préférentiellement les lymphocytes $\mathrm{V} \beta$ du lapin. Les cellules leucémogènes se distinguent des cellules non transformées par la présence, à leur surface, d'une glycoprotéine de $65 \mathrm{kDa}$. Cette protéine, dont la localisation normale est intracellulaire, peut être détectée à la surface des cellules transformées par un anticorps monoclonal. Il semblerait que la capacité leucémogène des cellules et l'expansion particulière des $V_{\beta 5}$ dépendent de la présence de cette protéine à la surface des cellules leucémogènes. Cela permet d'envisager que la transformation des cellules par le HTLV-l, et l'effet superantigénique qui lui est associé, s'accompagnent de la modification du routage, ou encore du démasquage, d'un superantigène cellulaire. A la lumière de ces résultats, il paraît souhaitable que les approches expérimentales qui tendent à identifier le superantigène potentiel du VIH parmi les protéines du virus soient reconsidérées.

\section{Le concept de superantigène se diversifie}

Les critères que doit remplir une protéine pour se démarquer d'un mitogène ou d'un antigène, et pour être considérée comme un superantigène, ont évolué. Tranchés au départ, ils laissent maintenant la place à quelques atténuations. Il est toujours établi que les superantigènes se distinguent des mitogènes car ils nécessitent la double présence de cellules $\mathrm{T}$ et de cellules stimulatrices porteuses de molécules du $\mathrm{CMH}$ de classe II. Il est toujours admis qu'ils induisent une stimulation polyclo- nés après exposition périnatale. En revanche, l'absence d'apprêtement antigénique par la cellule présentatrice n'est plus un critère obligé (par exemple: TSST-1*, MAM*, exotoxine A du Pseudomonas). Que la stimulation induite par un superantigène soit aussi un feu de paille et plonge la cellule dans un état de non-réponse n'est pas non plus une condition incontournable, et une fois de plus le TSST-1* se distingue, puisque, à la différence du staphylocoque de type A, par exemple, il n'induit pas d'anergie in vivo [25]. Il est en cela rejoint par le superantigène du virus de la rage qui ne semble pas induire d'état de nonréponse, mais qui, au contraire, présente la caractéristique remarquable de stimuler une réponse immune associée, quelle que soit la nature de l'antigène $[26,27]$.

Les superantigènes bactériens stimulent préférentiellement les cellules de type T helper 1 (Thl), ce qui proviendrait de leur capacité à induire la sécrétion de cytokines favorisant l'émergence de cette catégorie de lymphocytes [28, 29]. Ainsi, les superantigènes bactériens induiraient plutôt de l'IL2, de l'interféron $\gamma$ et du TGF- $\beta$ (transforming growth factor$\beta)$ [30]. Dans le cas des superantigènes stimulants, comme le MAM* ou la nucléocapside rabique, il peut être envisagé que la nature des cytokines produites sous leur impulsion soit totalement différente et aboutisse à la stimulation de lymphocytes de type Th2.

Nous constatons que la perception des superantigènes a rapidement évolué. Au début, imprégnés par le modèle des superantigènes bactériens dont les effets immunosuppresseurs étaient frappants, les immunologistes associaient communément au terme superantigène les notions d'anergie, de suppression, ou encore celle d'immunosuppression. Était alors oublié le premier effet d'un superantigène, qui est de stimuler fortement de façon polyclonale une ou plusieurs familles de lymphocytes $T$, quelle que soit leur spécificité antigénique, à la seule condition qu'ils expriment la bonne chaîne
$\mathrm{V}_{\beta}$. Nous assistons aujourd'hui avec les superantigènes viraux à l'installation d'une vision différente. Ainsi pour les MMTV*, le virus de la rage et même pour le VIH, c'est l'effet stimulateur massif qui est mis en avant. On peut, de plus, commencer à entrevoir, et le superantigène du virus de la rage en est certainement le pionnier, que certains superantigènes puissent aussi engendrer des relations T-B efficaces et s'afficher comme des immunostimulants. On s'achemine ainsi vers la reconnaissance de différentes sortes de superantigènes. Il y aurait les superantigènes bactériens, stimulateurs puissants, caractérisés par une activation des Thl et son corollaire de cytokines, se passant de molécules accessoires, puis des superantigènes de force intermédiaire, comme les superantigènes rétroviraux murins et le TSST-1*, et finalement des superantigènes faibles, comme le MAM* et la nucléocapside rabique pour les quels la présence de molécules accessoires, dont la molécule CD4, serait nécessaire à la consolidation de l'association TcR-CMH classe II. Ces superantigènes se démarqueraient des précédents par leur capacité à stimuler les Th2. Cela pourrait expliquer leur aptitude à induire des relations $\mathrm{T}-\mathrm{B}$ aboutissant à la production d'anticorps. Les superantigènes de cette dernière catégorie pourraient alors être utilisés comme des stimulants de réponses immunes.

Il reste à établir sur quelles bases moléculaires et sous quelles influences (nature des cellules présentatrices, des molécules accessoires) ces trois catégories de superantigènes sont capables d'engager les lymphocytes $T$ sur des voies d'activation différentes.

\section{Conclusion}

Il peut paraître surprenant de présenter un superantigène tantôt comme un activateur des lymphocytes $T$, tantôt comme un agent bloquant leur réponse. Ces deux propriétés caractérisent effectivement la

\footnotetext{
* Voir glossaire.
} 
dualité fonctionnelle des superantigènes dont l'impact sur la réponse immune se modifie au cours du temps. Il induit, tout d'abord, une prolifération intense des lymphocytes, puis, selon sa rémanence, une anergie transitoire ou permanente. L'état de non-réponse sera probablement entretenu par l'exposition répétée au superantigène. C'est ce qui se produirait lors d'infections chroniques (infections persistantes ou abortives installées par la vaccination rougeole à fort titre ou le VIH). Le superantigène aurait, en revanche, un effet stimulateur sur la réponse immune à un antigène associé, parce que le contact avec le superantigène serait ponctuel et l'antigène serait rencontré dans la phase proliférative. Et dans le cas d'infections où les réservoirs du virus seraient stimulés par le superantigène, il faciliterait l'invasion de l'hôte et donc l'infection virale. Vouloir définir qui, du virus ou de l'hôte, tire un avantage de la présence d'un superantigène, dépend certainement beaucoup de l'adhésion de chacun à une théorie de l'évolution. Les partisans du Darwinisme et de la pression sélective y seront plus enclins, ceux de la théorie neutraliste moléculaire émise par Motoo Kimura, pas du tout, - puisque le problème ne se pose pas -, et ceux qui suivront Stephen Gould introduisant la contingence et le hasard s'engageront dans le débat à l'occasion. L'importance de ce débat dépend en fait de la nature du superantigène, selon qu'il s'agit d'une protéine virale structurale ou d'une protéine accessoire ou induite. Il est, en effet, plus délicat de trouver une preuve de sélection naturelle sur la persistance d'un superantigène viral dont la fonction est avant tout d'être une protéine constitutive, que de se demander pourquoi une protéine non structurale est conservée. Ces réflexions ne pourront être profitablement engagées qu'après la découverte de nouveaux superantigènes. Dorénavant, les travaux entrepris sur les superantigènes viraux permettent d'éclairer d'un jour nouveau l'étude des rela- tions virus-hôtes et de proposer des mécanismes expliquant les manifestations immunopathologiques qui leur sont associées

\section{GLOSSAIRE}

$E B V$ : virus d'Epstein-Barr.

$H T L V-1$ : human T-lymphotrophic virus type 1.

MAM : mycoplasma arthritis mitogen.

MMTV: virus des tumeurs mammaires (mouse mammary tumor virus).

3'ORF : phase de lecture ouverte dans les séquences répétées du MMTV (3' open reading frame).

TSST-1 : toxic shock syndrome toxin type 1 .

$V_{\beta}$ : région variable de la sous-unité $\beta d u$ récepteur $T$. Site d'interaction avec les superantigènes.

VIH: vinus de l'immunodéficience humaine.

\section{RÉFÉRENCES}

1. Marrack P, Kappler J. The staphylococcal enterotoxins and their relatives. Science 1990 ; 248 : 705-11.

2. Cole BC, Atkins CL. The Mycoplasma arthritis $\mathrm{T}$ cell mitogen, MAM : a model superantigen. Immunol Today 1991; 12: 271-6.

3. Huber BT. Mls genes and selfsuperantigens. Trends Genet 1992; 8: 399-402.

4. Acha-Orbea H, Held W, Waanders GA, Shakhov AN, Scarpellino L, Lees RK, MacDonald HR. Exogenous and endogenous mouse mammary tumor virus superantigens. Immunol Rev 1993; 131: 5-25.

5. Lafon M, Lafage M, Martinez-Arends A, Ramirez R, Vuillier F, Charron D, Lotteau $\mathrm{V}$, Scott-Algara, D. Evidence in humans of a viral superantigen. Nature 1992; 358 : 507-10.

6. Taylor AH, Haberman AM, Gerhard W, Caton AJ. Structure-function relationships among highly diverse $T$ cells that recognize a determinant from influenza virus hemagglutinin. J Exp Med 1990; 172: 1643-51.

7. Aebisher $\mathrm{T}$, Oehen $\mathrm{S}$, Hengartner $\mathrm{H}$. Preferential usage of $\mathrm{V}_{\alpha} 4$ and $\mathrm{V}_{\beta} \mathrm{I} 0 \mathrm{~T}$ cell receptor genes by lymphocytic choriomeningitis virus glycoprotein-specific $\mathrm{H}-2 \mathrm{Db}$-restricted cytotoxic T cells. Eur J Immunol 1990; 20 : 523-31.
8. Paillard X, West SG, Iafferty JA, Clements JR, Kappler JW', Marrack P, Kotzin BL. Evidence for the effects of a superantigen in rheumatoid arthritis. Saience 1991 ; 253 : 325-9.

9. Dalgleish AG, Wilson S, Gompels M, Ludlam C, Gazzard B, Coates AM, Habeshaw J. T-cell receptor variable gene products and early HIV-1 infection. Iancet 1992; 339: 824-8.

10. Smith TJ, Terada N, Robinson CC, Gelfand EW. Acute infectious mononucleosis stimulates the selective expression/expansion of $\mathrm{V}_{\beta}, 6.1-3$ and $\mathrm{V}_{\beta 7} \mathrm{~T}$ cells. Blood $1993 ; 81$ : 1521-6.

11. Held W, Shakhov AN, Izui S, Waanders GA, Scarpellino L, MacDonald HR, Acha-Orbea H. Superantigen-reactive $\mathrm{CD}^{+} \mathrm{T}$ cells are required to stimulate $\mathrm{B}$ cells after infection with mouse mammary tumor virus. J Exp Med 1993; 177 : 359-66.

12. Laurence J, Hodtsev AS, Posnett D. Superantigen implicated in dependence of HIV-1 replication in $T$ cells on $T c R V_{\beta}$ expression. Nature 1992; 358 : 255-9.

13. Pantaleo G, Graziosi C, Fauci A. The immunopathogenesis of human immunodeficiency virus infection. $N$ Engl J Med $1993 ; 328: 327-35$

14. Posnett DN, Kabak S, Hodtsev AS, Goldberg EA, Asch A. T-cell antigen receptor $V_{B}$ subsets are not preferentially deleted in AIDS. AIISS 1993; 7 : 625-31.

15. White J, Herman A, Pullen AM, Kubo R, Kappler JW, Marrack $P$. The $V_{\beta}$-specific superantigen staphylococcal enterotoxin B : stimulation of mature $T$ cells and clonal deletion in neonatal mice. Cell 1989; 56 : 27-35.

16. Marrack P, Kushnir E, Kappler J. A maternally inherited superantigen encoded by a mammary tumour virus. Nature 1991 ; 349 : $524-6$.

17. Imberti L, Sottini A, Bettinardi A, Puoti M, Primi D. Selective depletion in HIV infection of $T$ cells that bear specific $T$ cell receptor $V_{\beta}$ sequences. Science $1991 ; 254: 860-2$.

18. Hodara VL, Jeddi-Tehrani M, Grunewald J, Andersson R, Scarlatti G, Esin G, Holmberg V, Libonatti O, Wigzell H. HIV infection leads to differential expression of $T$-cell receptor $V_{\beta}$ genes in $\mathrm{CD}^{+}$and $\mathrm{CD}^{+}{ }^{+} \mathrm{T}$ cells. AIIS $1993 ; 7: 633-8$.

19. Soudeyns H, Pantaleo GP, Boghossian T, Ciurli C, Rebai N, Lane HC, Fauci AS, Sekaly RP. $V_{\beta}$-specific variations in the TCR repertoire of HIV-infected subjects suggest the presence of an HIV-associated superantigen. Joint Meeting of the American Association of Immunologists and the Clinical Immunology Society. J Immunol 1993; 150: 104A. 
20. Janeway (iA. Immune recognition: Mlsmakes a little sense. Vature 1991 ; 349: 459-61.

21. Mosier IDE, (;ulizia RI, MacIsaac PI), Torbett BE, Levy JA. Rapid loss of (I) $4^{+} \mathrm{T}$ cells in human-PBL-SCII) mice by noncytopathic HII' isolates. Science 1993; 260): 689-92.

22. (iarenne M, I eroy (), Beau JP. (hild mortality after high-titre measles vaccination : a protective study in Senegal. Inncet $1991 ; 338: 903-7$

23. Korman AJ, Bourgarel $P$, Meo $T$, Rieckhof (;E. The mouse mammary tumour virus long terminal repeat encodes a type Il transmembrane glycoprotein. EMBO / 1992; 11: 1901-5.

24. Seto A, Kumagai K. I eukemogenesisassociated antigen in HTLV-l transformed rabbit cells. Ieukemia Res 1993; 17 : 567-72.

25. ()chi A, Yuh K, Migita K. Not every superantigen induces tolerance in vivo. Semin Immunol 1993: 5: 57-63.

26. Dietzschold B, W'ang H, Rupprecht (E, Celis E, Tollis M, Frtl H, Heber-Katz $\mathrm{E}$, Koprowski H. Induction of protective immunity against rabies by immunization with rabies virus ribonucleoprotein. Pros Natl Arad Sri USA 1987; 84: 9165-9.

27. Herzog M, Lafage M, Montano-H lirose JA, Frivell (:, Scott-Âlgara I), I afon M. NC: specific $T$ and $B$ cell responses in humans after rabies vaccination. Vinus Res 1992; 24 : 77-89.

28. Schmitz J, Radbruch A. Iistinct antigen presenting cell-derived signals induce Th cell proliferation and expression of effector cytokines. Int Immunol 1991; 4: 4.3-51.

29. Cardell S, Höidén I, Möller (;. Manipulation of the superantigen-induced lymphokine response. Selective induction of interleukin-I() response or interferongamma synthesis in small resting (.I) $4^{+} \mathrm{T}$ cells. Eur / Immunol 1993; 23 : 523-9.

30. Nagelkerken L, (ollob KJ, Tielemans M. Coffman RL. Role of transforming growth factor- $\beta$ in the preferential induction of T-helper cells of type 1 by staphylococcal enterotoxin B. Eur J Immunol $1993 ; 23: 2306-10$.

\section{Monique Lafon}

Institut Pasteur, département de virologie, 25, rue du Docteur-Roux, 75724 Paris Cedex 15, France.

\section{TIRÉS A PART}

M. Iafon. 